\title{
Transient simulation of electrochemical machining processes for manufacturing of surface structures in high-strength materials
}

\author{
Sascha Loebel ${ }^{1}$, Mike Zinecker ${ }^{1}$, Philipp Steinert ${ }^{1}$, and Andreas Schubert $^{1}$ \\ ${ }^{1}$ Chemnitz University of Technology
}

August 4, 2020

\begin{abstract}
Electrochemical machining (ECM) is a method for removing metal by anodic dissolution. At the interface between the workpiece surface and an electrically conductive fluid (electrolyte), the material is dissolved locally without direct physical contact to the cathodic tool. Due to the force-free nature of the process, ECM is used for machining high-strength or hard materials, such as titanium aluminides, Inconel, Waspaloy, and high nickel, cobalt, and rhenium alloys.1 However, determining suitable process parameters remains challenging due to their interacting effects on working distances during the machining process. Therefore a simulation-based approach to process design substantially reduces resource and time investment to achieve the desired geometry of the finished part. This methodology requires data about the materials electrochemical properties, such as removal velocity and current efficiency, which have to be obtained experimentally. In this study, a methodology for acquiring and processing this data as well as the development of multiphysics simulation models is presented for two use cases: (i) manufacturing a centrifugal impeller with a diameter of $14 \mathrm{~mm}$ consisting of the nickel alloy Inconel 713C for use in turbomachinery and (ii) the generation of a defined surface micro structure into the novel Mg-Y-Zn alloy WZ73.
\end{abstract}

\section{Hosted file}

AMARETO_Loebel_Simulation_ECM.pdf available at https://authorea.com/users/348676/articles/ 473884-transient-simulation-of-electrochemical-machining-processes-for-manufacturing-ofsurface-structures-in-high-strength-materials

\section{Hosted file}

build_files.zip available at https://authorea.com/users/348676/articles/473884-transientsimulation-of-electrochemical-machining-processes-for-manufacturing-of-surfacestructures-in-high-strength-materials 